\title{
GENETIC VARIATION IN DEFENSE CHEMISTRY IN WILD CABBAGES AFFECTS HERBIVORES AND THEIR ENDOPARASITOIDS
}

\author{
Rieta Gols, ${ }^{1,4}$ R. Wagenaar, ${ }^{2}$ Tibor Bukovinszky,${ }^{1}$ Nicole M. van Dam, ${ }^{2}$ Marcel Dicke, ${ }^{1}$ James M. Bullock,${ }^{3}$ \\ AND JefFrey A. Harvey ${ }^{2}$ \\ ${ }^{1}$ Laboratory of Entomology, Department of Plant Sciences, Wageningen University, The Netherlands \\ ${ }^{2}$ Department of Multitrophic Interactions, Netherlands Institute of Ecology, Center for Terrestrial Ecology, Heteren, The Netherlands \\ ${ }^{3}$ Center for Ecology and Hydrology, Crowmarsh Gifford, Wallingford, United Kingdom
}

\begin{abstract}
Populations of wild Brassica oleracea L. grow naturally along the Atlantic coastlines of the United Kingdom and France. Over a very small spatial scale (i.e., $<15 \mathrm{~km}$ ) these populations differ in the expression of the defensive compounds, glucosinolates (GS). Thus far, very few studies have examined interactions between genetically distinct populations of a wild plant species and associated consumers in a multitrophic framework. Here, we compared the development of a specialist (Pieris rapae) and a generalist (Mamestra brassicae) insect herbivore and their endoparasitoids (Cotesia rubecula and Microplitis mediator, respectively) on three wild populations and one cultivar of $B$. oleracea under controlled greenhouse conditions. Herbivore performance was differentially affected by the plant population on which they were reared. Plant population influenced only development time and pupal mass in $P$. rapae, whereas plant population also had a dramatic effect on survival of $M$. brassicae. Prolonged development time in $P$. rapae corresponded with high levels of the indole GS, neoglucobrassicin, whereas reduced survival in $M$. brassicae coincided with high levels of the aliphatic GS, gluconapin and sinigrin. The difference between the two species can be explained by the fact that the specialist $P$. rapae is adapted to feed on plants containing GS and has evolved an effective detoxification system against aliphatic GS. The different $B$. oleracea populations also affected development of the endoparasitoids. Differences in foodplant quality for the hosts were reflected in adult size in C. rubecula and survival in $M$. mediator, and further showed that parasitoid performance is also affected by herbivore diet.
\end{abstract}

Key words: bottom-up effects; Brassica oleracea; coevolution; Cotesia rubecula; glucosinolates; Mamestra brassicae; Microplitis mediator; Pieris rapae; plant defense; top-down effects.

\section{INTRODUCTION}

Plant defense strategies against insect herbivores and pathogens often involve the production of allelochemicals (secondary plant metabolites), which are usually phylogenetically conserved in specific plant families or genera (e.g., coumarins in Umbelliferae, tropane alkaloids in Solanaceae, and glucosinolates in the Brassicaceae; Rosenthal and Berenbaum 1992, Rask et al. 2000, Schoonhoven et al. 2005). A vast amount of literature has demonstrated that many secondary plant compounds are active against insect herbivores. These compounds can act as feeding deterrents or significantly alter the physiology and development of some herbivores, through reduced growth rates, smaller adult size, and increased mortality (Zangerl and Berenbaum 1993, Li et al. 2000, van Dam et al. 2000). Conversely, many specialist herbivores are known to exhibit resistance to secondary plant compounds and may require them as feeding stimulants (Bowers 1983, Renwick 2002,

Manuscript received 25 May 2007; revised 17 August 2007; accepted 21 September 2007; final version received 19 October 2007. Corresponding Editor: K. F. Raffa.

${ }^{4}$ E-mail: rieta.gols@wur.nl
Schoonhoven et al. 2005). Some resistant herbivores sequester plant toxins in their hemolymph or body tissues, thus providing them with some degree of protection from their natural enemy complex (Nishida 2002).

Insect herbivore populations are not only affected by plant characteristics (bottom-up effects), but also by the presence of natural enemies such as predators and parasitoids (top-down effects). The relative strength of bottom-up and top-down control of insect herbivores in natural ecosystems has been the subject of debate over many years (Hairston et al. 1960, Keeler et al. 2006, Gripenberg and Roslin 2007). Many studies examining the effects of plant defense on insect herbivores have either focused exclusively on interactions between plants and insect herbivores (direct defense) or between plants and natural enemies of insect herbivores (indirect defense). Secondary plant compounds have been shown to negatively affect the development of organisms in the third (Campbell and Duffey 1979, Barbosa et al. 1991, Roth et al. 1997) and even the fourth (Orr and Boethel 1986, Harvey et al. 2003, 2007) trophic level. On the other hand, prolonged development of the host on poorquality host plants may extend the window of vulnerability to parasitism (the "slow growth-high mortality 
hypothesis"; sensu Benrey and Denno 1997, Williams 1999). These results suggest that top-down and bottomup control of insect herbivores may not be simple alternatives and that we may need to acknowledge complex interactions between top-down and bottom-up effects that vary over spatial and temporal gradients (Gripenberg and Roslin 2007).

Generalist insects are generally much more strongly affected by plant secondary chemistry than specialists (Blau et al. 1978, Giamoustaris and Mithen 1995). Therefore, to better understand the effects of interpopulation variation in plant defenses on higher trophic level interactions, these interactions should be studied for generalists and specialists separately. Because some defensive traits are inducible (see Karban and Baldwin 1997), induction patterns of defensive traits in response to specialist and generalist herbivores should also be considered.

Populations of wild cabbage, B. oleracea L. (Brassicaceae), grow naturally along the Atlantic coastlines of the United Kingdom and France (Mitchell and Richards 1979). Previous studies have reported differences in the expression of glucosinolates (hereafter GS) among populations of $B$. oleracea over a limited spatial scale (Mithen et al. 1995, Moyes et al. 2000, Moyes and Raybould 2001). The expression of GS is under control of several genes (Magrath et al. 1993, Halkier and Gershenzon 2006) and is highly heritable (Mithen et al. 1995). Raybould et al. (1999) have demonstrated that the Dorset populations of wild B. oleracea are genetically differentiated. Since these populations have evolved under natural selection pressures, differences between the populations are most likely caused by variation in local biotic and abiotic characteristics. For instance, some of the Dorset populations of B. oleracea are found on exposed cliff sides, whereas others grow in more secluded areas. This profoundly affects their degree of exposure to the prevailing wind, which in turn may hinder the ability of flying insects to either colonize the plants, or else influences the dispersal of plantrelated odors that are used by insects to locate plants among other stands of interstitial vegetation. However, random processes, such as genetic drift, may also play a role in shaping differences in GS chemistry.

Herbivore densities on different populations of wild B. oleracea in Dorset have been shown to vary, although densities of specialist herbivores were not correlated with measured levels of GS compounds (Moyes et al. 2000). In the study by Moyes et al. (2000), herbivore densities were measured over two consecutive years, but samples for GS were only taken once. (Higher trophic levels were not included in the survey.) GS levels have been shown to fluctuate over the course of a growing season and tend to be higher in the fall than in the summer (Agerbirk et al. 2001, Gols et al. 2007). Furthermore, GS concentrations change in response to herbivore feeding damage (Bodnaryk 1992, Agrawal et al. 2002, Traw and Dawson 2002). Therefore, GS concentrations in natural populations are the combined result of constitutive and induced levels as well as seasonal fluctuations. To link levels of GS to performance of herbivores in the field, both constitutive and induced levels of GS need to be separated (but see Traw 2002).

This study examines growth and development of two herbivorous insect species, Pieris rapae L. (Lepidoptera: Pieridae) and Mamestra brassicae L. (Lepidoptera: Noctuidae), when reared on three of the wild Dorset populations and a cultivar of $B$. oleracea under controlled greenhouse conditions. Both herbivore species are among the most serious pests of cultivated cabbage over much of Europe, but may also attack other related wild crucifers (Theunissen et al. 1985). The cultivar served as a population with low levels of GS in leaf tissue compared to the wild populations. P. rapae is a specialist herbivore on brassicaceous plant species, whereas $M$. brassicae is a generalist insect herbivore, which feeds on plants in several plant families including the Brassicaceae (Carter 1984). Furthermore, we investigated the effect of food-plant quality mediated through the host on the performance of two closely related solitary endoparasitoids of the two herbivores, Cotesia rubecula Marshall (Hymenoptera: Braconidae) and Microplitis mediator Halliday (Hymenoptera: Braconidae). In addition we measured levels of GS in plants damaged by herbivores and determined if they correlated with insect performance. We predict that differences in GS levels among the different B. oleracea populations are reflected in the development and survival of the insect herbivores and their parasitoids, but that the development of the generalist and its parasitoid is more negatively affected than the better adapted specialist and its parasitoid.

\section{Methods \\ Plants}

Brassica oleracea seeds were collected from several plants $(>15)$ in three wild populations growing within 10 $\mathrm{km}$ distance on chalk cliffs along the south coast of the United Kingdom, near Swanage in Dorset, in September 2005. The three wild populations were located at sites known as "Old Harry" $\left(50^{\circ} 38^{\prime} \mathrm{N}, 1^{\circ} 55^{\prime} \mathrm{E}\right)$, "Kimmeridge" $\left(50^{\circ} 35^{\prime} \mathrm{N}, 2^{\circ} 03^{\prime} \mathrm{E}\right)$, and "Winspit" $\left(50^{\circ} 36^{\prime} \mathrm{N}, 2^{\circ} 07^{\prime}\right.$ E). Hereafter the different populations will be referred to as $\mathrm{OH}$ (Old Harry), KIM (Kimmeridge), and WIN (Winspit). As a reference plant population, we used a cultivated variety of B. oleracea, gemmifera (Brussels sprout) cv. Cyrus (hereafter CYR), because it contains low levels of GS compared to the wild Dorset populations of B. oleracea in England (Moyes et al. 2000, Gols et al. 2007).

Seeds were germinated and seedlings were subsequently transferred to $2-\mathrm{L}$ pots containing a soil mixture of $30 \%$ sand, $5 \%$ clay, and $65 \%$ peat. Plants were grown in a greenhouse at $20-30^{\circ} \mathrm{C}, 40-80 \%$ relative humidity, and a photoperiod of at least $16 \mathrm{~h}$. If the light dropped 
below $500 \mu \mathrm{mol}$ photons $\cdot \mathrm{m}^{-2} \cdot \mathrm{s}^{-1}$ during the $16-\mathrm{h}$ photoperiod, supplementary illumination was applied by high-pressure mercury lamps. Plants were watered daily. When the plants were four weeks old, they were fertilized once a week with $100 \mathrm{~mL} 0.5$ Hoagland solution, which was applied to the soil. Plants were eight weeks old when they were used in experiments. Fertilizing and watering were continued during the experiments.

\section{Insects}

The insect herbivores and parasitoids used in this study are native to the Palaearctic region and sympatric in their overall distributions. All insects, with the exception of Microplitis mediator, were originally collected from a Brussels sprout field in the vicinity of Wageningen, The Netherlands, and were maintained on Brussels sprouts in the laboratory. Cocoons of $M$. mediator were obtained from a culture maintained at Agriculture Canada Laboratories in Saskatoon, Saskatchewan, Canada. Cotesia rubecula is a highly specialized solitary endoparasitoid that only attacks first and second instar larvae (hereafter, L1-L2) of Pieris rapae (Harvey et al. 1999). Parasitized host larvae were reared on Brussels sprout until the parasitoids had completed larval development and egressed from the host. M. mediator is a fairly specialized solitary endoparasitoid that attacks L1-L4 instar larvae of $M$. brassicae and a few closely related hosts in the moth family Noctuidae (Arthur and Mason 1986). In this study $M$. mediator was reared on $M$. brassicae. Newly hatched L1 instar larvae obtained from the general $M$. brassicae culture were reared on an artificial insect diet (ingredients per liter: $28 \mathrm{~g}$ agar, $160 \mathrm{~g}$ corn flower, $50 \mathrm{~g}$ beer yeast, $50 \mathrm{~g}$ wheat germ, $2 \mathrm{~g}$ sorbic acid, $1.6 \mathrm{~g}$ methyl-4-hydroxybenzoate, $8 \mathrm{~g}$ ascorbic acid, $0.5 \mathrm{~g}$ streptomycin, and water). When the larvae had developed into L2, they were parasitized by mated $M$. mediator females in large petri dishes. Parasitized caterpillars were reared on the artificial diet until the parasitoid larvae egressed from the host and pupated. Parasitoid cocoons were collected and transferred to a new cage ( $C$. rubecula) or petri dish ( $M$. mediator). Newly emerged adults were provided with honey and water, and mated females were used in experiments approximately five days after adult emergence. All insects used in this study were reared in climate rooms or greenhouses at $22^{\circ} \pm 2^{\circ} \mathrm{C}, 50-70 \%$ relative humidity, with a photoperiod of at least $16 \mathrm{~h}$.

\section{Glucosinolate analysis}

To determine GS levels in leaf tissues of the different $B$. oleracea populations, samples were taken from undamaged control plants $(n=5)$ and from plants damaged by $P$. rapae whether the caterpillars were either parasitized $(n=9)$ or unparasitized $(n=6)$. The plants that were used in the performance bioassay (see Methods: Insect performance) were also used for tissue sampling. Samples were taken from plants that had been damaged by $P$. rapae caterpillars for seven days. Undamaged control plants were of the same age and were kept in the same greenhouse. A second cohort of undamaged plants was sampled two weeks later to establish the relationship between GS content and survival of $M$. brassicae (see Methods: Statistical analysis). Two discs, $7 \mathrm{~mm}$ in diameter, were punched per leaf, one from each central part of a leaf half, and six fully unfolded leaves were sampled per plant. Leaf discs were pooled per plant in 2-mL Eppendorf vials and frozen in liquid nitrogen immediately after sampling. Samples were later freeze-dried and pulverized. Aliquots of dried material $(50 \mathrm{mg})$ were weighed in $2-\mathrm{mL}$ centrifuge tubes. GS were extracted and purified as described in van Dam et al. (2004), which should be consulted for a more detailed description. GS were separated on a reverse-phase C-18 column (Alltima C$18,3 \mu \mathrm{m}, 150 \times 4.6 \mathrm{~mm}$; Alltech, Deerfield, Illinois, USA) on HPLC (DIONEX, Sunnyvale, California, USA) with an acetonitrile water gradient. Detection was performed with a DIONEX PDA-100 Photodiode array detector set to scan from $200 \mathrm{~nm}$ to $350 \mathrm{~nm}$. For quantification, sinigrin (Sigma, St. Louis, Missouri, USA) was used as an external standard. Peaks were integrated at $229 \mathrm{~nm}$ for which standard response factors have been defined (European Community 1990). The different GS were identified based on their retention times and UV spectra compared to those of the pure compounds, sinigrin and glucobrassicin, or compared with a certified oil seed reference (EC Community Bureau of Reference BCR-367R, Fluka, Buchs, Switzerland).

\section{Insect performance}

To investigate whether variation in plant chemistry affects higher trophic levels, the different insect herbivores and their parasitoids were reared on plants from the four different $B$. oleracea populations. Experiments were conducted in two greenhouses under the same conditions as for the plants. We used a separate greenhouse for each herbivore-parasitoid combination.

Specialist plant association: Pieris rapae and Cotesia rubecula.-To obtain plants with eggs of $P$. rapae, one plant from each population was placed in the rearing cage with adult $P$. rapae butterflies for four hours. Plants were then removed from the cage and left in the rearing room for the eggs to hatch and the larvae to develop until they reached L2. For each plant population one cohort of 54 unparasitized larvae, which served as a control, was transferred and divided over six new plants of each population. A second cohort of 100 larvae was parasitized by C. rubecula. For parasitism, an individual mated female parasitoid was presented with a single L2 $P$. rapae larva. A larva was considered to be parasitized when the female inserted into and removed her ovipositor from the host. Each parasitoid female was allowed to parasitize up to a maximum of 10 larvae. 
Parasitized larvae were evenly distributed over nine new plants of the population on which the unparasitized larvae had been feeding previously.

Caterpillars were allowed to develop and move freely on the plants until pupation of the herbivore or egression of the parasitoid larva from the host and subsequent pupation. Unparasitized caterpillars tend to wander and leave the food plant in the final phase of the larval development to search for a site to pupate. Therefore, unparasitized control larvae in the late final (fifth) instar were transferred to plastic containers $(15 \times$ $12 \times 6 \mathrm{~cm}$ ) containing some excised leaf material. Larvae were checked twice a day and when they had pupated, the date of pupation was recorded and the pupae were weighed on a Cahn microbalance (Cahn Instruments, Cerritos, California, USA; accuracy $1 \mu \mathrm{g}$ ).

Parasitoid cocoons were collected from the plant and kept individually in labeled vials until adult emergence. When the adult wasps emerged, the date of eclosion was recorded and their sex was determined visually (the ovipositor of female wasps is visible on the abdomen). Wasps were anesthetized with $\mathrm{CO}_{2}$ and subsequently weighed on the Cahn microbalance. A subsample of male and female $C$. rubecula wasps was dried in an oven at $80^{\circ} \mathrm{C}$ for three days to establish the relationship between fresh and dry mass. Cocoons were checked several times daily for adult parasitoid emergence. As the time of oviposition, we used the time at which $50 \%$ of the larvae were parasitized, which took about one hour. Since the time of pupation of the herbivore could not be determined accurately, development time was measured in days.

Generalist plant association: Mamestra brassicae and Microplitis mediator.-Sheets with M. brassicae eggs were obtained from the general culture. These sheets were incubated until the eggs hatched. Newly hatched larvae were then evenly distributed among four plants, one plant of each $B$. oleracea population. When the larvae had reached L2, they were parasitized by $M$. mediator, using the method described for $P$. rapae and $C$. rubecula. Unfortunately, the number of $M$. brassicae larvae surviving on the different plant populations varied greatly. The highest number of larvae was recovered from CYR and KIM plants. In contrast, none of the larvae survived on WIN, which was therefore excluded from the parasitism experiment. To obtain sufficient larvae that were reared on $\mathrm{OH}$, we transferred some of the larvae that were initially reared on CYR to the $\mathrm{OH}$ population after parasitism. The final number of parasitized hosts on the different populations was 70 larvae on CYR and 100 larvae on both KIM and $\mathrm{OH}$. To ensure that the larvae did not leave their respective food plants, parasitized larvae were reared in plastic containers $(15 \times 12 \times 6 \mathrm{~cm})$ on excised leaves. Every two days survival was monitored, leaf material replaced by fresh leaves, and feces removed until the parasitoid egressed from the host. Parasitoid cocoons were collected and transferred to labeled glass vials. Development time and adult fresh mass were determined as for $C$. rubecula.

Because early survival of the $M$. brassicae larvae varied considerably when reared on intact plants from the different populations, development of healthy (i.e., unparasitized) larvae was monitored in more detail in a separate experiment. Newly hatched larvae (200 per plant population), were transferred in groups of 10 to small petri dishes $(9 \mathrm{~cm}$ diameter) with pieces of excised leaves. Every two days survival was monitored, leaf material replaced with fresh leaves, and feces removed. As the larvae grew and molted to L3, they were moved to larger petri dishes (13 $\mathrm{cm}$ diameter) and, in the final instar (L5), to plastic containers $(15 \times 12 \times 6 \mathrm{~cm})$. To reduce the number of plants required, the number of individuals was reduced to $\sim 100$ on CYR, KIM, and $\mathrm{OH}$ plants when the caterpillars reached L3. At this point, the number of remaining caterpillars on WIN plants was already $<100$ individuals. Petri dishes were randomly selected from each population treatment and discarded. When the caterpillars reached L5, containers were lined with a mixture of soil and vermiculite in preparation for pupation, which in M. brassicae occurs belowground. This took approximately one week. Pupae were collected daily and their fresh mass was measured immediately.

\section{Statistical analysis}

Development time from egg to pupa and pupal mass of individual $P$. rapae and $M$. brassicae reared on the different plant populations were analyzed using a general linear model ANOVA with plant population as main factor. Similarly, we used a general linear model ANOVA to compare development time from egg to adult and adult body mass of individual C. rubecula. Here we included plant population and sex as well as their interaction as factors in the model. To compare levels of glucosinolates (GS) a general linear model ANOVA was used on ln-transformed GS concentrations in individual plants with plant population, plant treatment (undamaged control, damaged by unparasitized $P$. rapae, and damaged by parasitized $P$. rapae), and plant population $\times$ treatment interaction as factors. Post hoc Tukey-Kramer multiple comparisons tests were conducted when the ANOVA models were significant. Analyses were carried out using SAS software package 8.02 (SAS Institute 1999-2001).

Data on the survival of $M$. brassicae were analyzed using nonparametric Kaplan-Meier survival curves (Kleinbaum 1996). Data were censored to account for the reduction in number of larvae on plants. The logrank test was used to compare the survival curves on the different plant populations and a sequential Bonferroni correction was applied to adjust $\alpha$ for multiple comparisons.

Linear least-squares regression analysis was carried out to establish the relationship between mean concentrations of GS compounds in the different plant 


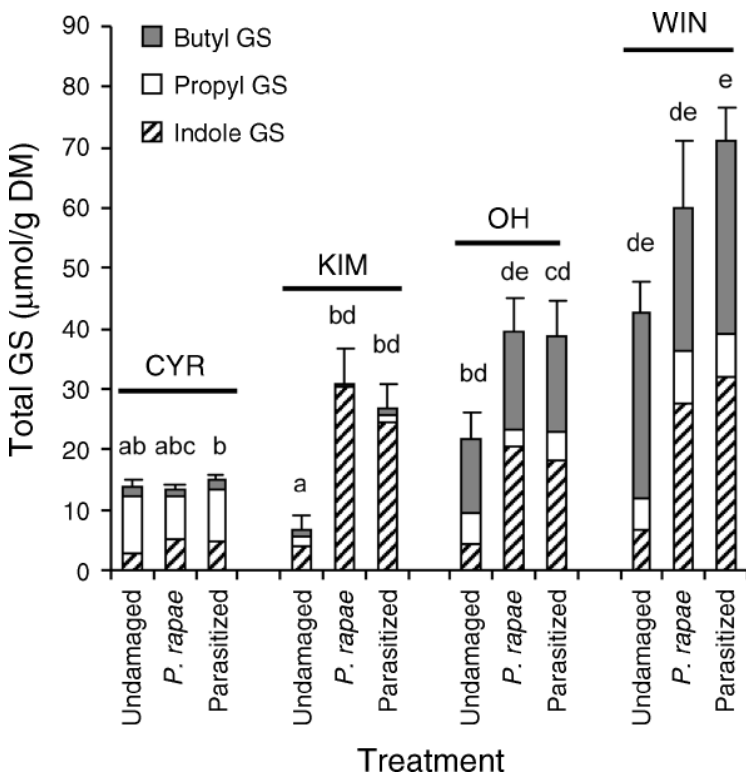

FIG. 1. Total glucosinolate (GS) concentrations in leaf tissues (mean $+\mathrm{SE})$ in a cultivar (CYR) and in each of three wild populations (KIM, OH, and WIN) of Brassica oleracea from the United Kingdom (Dorset), when reared under standardized greenhouse conditions. GS quantities were measured in leaf tissue sampled from undamaged plants, plants damaged by unparasitized Pieris rapae larvae, and plants damaged by $P$. rapae larvae that were parasitized by Cotesia rubecula. Total glucosinolate levels were divided into aliphatic GS derived from a propyl (IBE and SIN; see Fig. 2 for the full names) and a butyl side chain (RAPH, GNA, and PRO), and indole GS (GBC, NEO, 4OH, 4MeOH). Bars with the same letter are not significantly different (Tukey-Kramer test for multiple comparisons among means with $\alpha=0.05$ )

populations and mean values of the different fitness parameters (development time, adult body mass, survival) of the herbivores when reared on these plant populations. For the analysis, we used GS concentrations measured in plant tissue when the larvae were in the third larval instar. M. brassicae was reared on detached leaves; therefore, we used GS data of undamaged plants that were sampled in the third week since oviposition. For P. rapae we used the GS concentrations measured in plants that were fed upon by $P$. rapae for seven days.

\section{RESULTS \\ Glucosinolates}

Glucosinolate analyses of samples taken from leaf tissues revealed considerable quantitative variation between the four B. oleracea populations (Figs. 1 and 2). In all plant populations the same nine different glucosinolates (GS) were detected except for neoglucobrassicin in CYR plants; however, previous work detected low levels of this compound in the same cultivar (Gols et al. 2007). The following GS were found: two propyl-derived GS (glucoiberin and sinigrin), three butyl-derived GS (progoitrin, gluconapin, and glucoraphanin), and four indole GS (glucobrassicin, neoglucobrassicin, 4-hydroxyglucobrassicin, and 4-methoxyglucobrassicin). Plant population had a significant effect on levels of all individual GS except for progoitrin $\left(F_{3,62}=1.75, P=0.17\right)$ and 4-hydroxyglucobrassicin $\left(F_{3,62}=1.76, P=0.16\right)$.

Larval feeding damage had a significant effect on concentrations of neoglucobrassicin $\left(F_{2,62}=19.8, P<\right.$ $0.001)$ and glucobrassicin $\left(F_{2,62}=32.0, P<0.001\right)$, but not on the other GS. For glucobrassicin the interaction between plant population and treatment was also significant $\left(F_{6,62}=2.57, P=0.03\right)$. Only in the wild plant populations were glucobrassicin concentrations higher in damaged plants than in undamaged plants. In
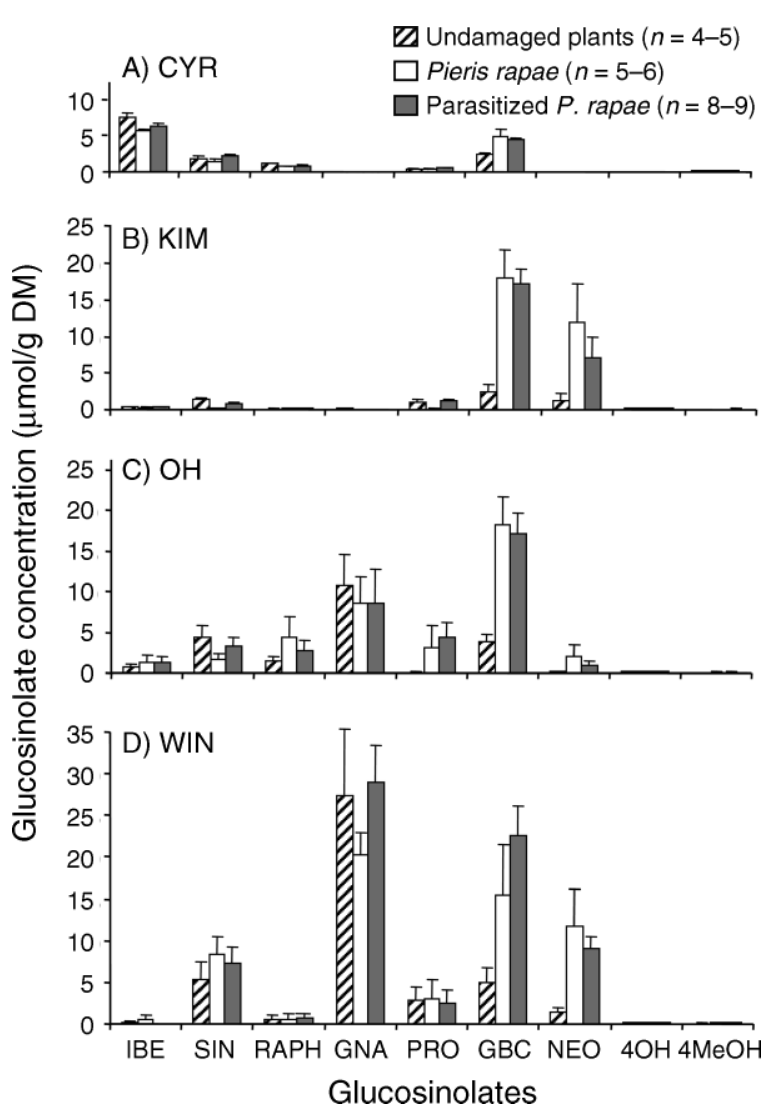

FIG. 2. Glucosinolate (GS) concentrations in leaf tissues (mean $+\mathrm{SE} ; \mu \mathrm{mol} / \mathrm{g}$ dry mass $[\mathrm{DM}])$ in (A) a cultivar (CYR), and in three different wild populations, (B) KIM, (C) $\mathrm{OH}$, and (D) WIN, of B. oleracea from the United Kingdom (Dorset), when reared under standardized greenhouse conditions. GS quantities were measured in leaf tissue sampled from undamaged plants (hatched bars), plants damaged by unparasitized Pieris rapae larvae (open bars), and plants damaged by parasitized $P$. rapae larvae (solid bars). Damaged plants were exposed to herbivore feeding for seven days, and all plants were of the same age when sampled. The following GS compounds were detected: glucoiberin (IBE), sinigrin (SIN), glucoraphanin (RAPH), gluconapin (GNA), progoitrin (PRO), glucobrassicin (GBC), neoglucobrassicin (NEO), 4-hydroxyglucobrassicin $(4 \mathrm{OH})$, and 4-methoxyglucobrassicin $(4 \mathrm{MeOH})$. 
WIN plants the difference between damaged and undamaged plants was only significant for plants damaged by parasitized $P$. rapae caterpillars. The effect of induction was most pronounced in the KIM population, where damage resulted in a fourfold increase of total GS, whereas there was little change in the CYR plants (Fig. 1). Parasitized and unparasitized caterpillars induced the plants in a similar way (pairwise comparisons were never significantly different).

The relative contribution of the different GS to the total GS profile differed among populations and between damaged and undamaged plants (Figs. 1 and 2). In undamaged plants, glucoiberin was the dominant compound in CYR plants (54\% of total), whereas in $\mathrm{OH}$ and WIN the GS profile was dominated by gluconapin (50\% and $64 \%$ of total, respectively). In undamaged KIM plants, neoglucobrassicin and sinigrin comprised $20 \%$ and $17 \%$ of the GS content, respectively. In plants that were induced by unparasitized or parasitized $P$. rapae indole GS dominated the GS profile. In the KIM plants, indole GS contributed $>90 \%$ of the total GS content in induced plants, compared to between 39\% and $52 \%$ in the other three populations.

\section{Performance studies}

Pieris rapae-Cotesia rubecula.-All plant populations were readily accepted for oviposition by female $P$. rapae butterflies. Females laid $>150$ eggs on each plant from the four different populations within a 4-h oviposition period. Plant population had a significant effect on pupal mass $\left(F_{3,183}=5.0, P=0.002\right.$; Fig. $\left.3 \mathrm{~A}\right)$ and development time from egg to pupa $\left(F_{3,183}=44.6, P<\right.$ 0.001; Fig. 3B). Pupal mass was significantly higher when the larvae were reared on $\mathrm{CYR}$ and $\mathrm{OH}$, compared to KIM. Pupae reared on WIN plants were of intermediate mass. Larvae developed the fastest on the populations on which they attained the highest pupal mass (i.e., $\mathrm{CYR}$ and $\mathrm{OH}$; Fig. 3). The development time from egg to pupa was longest on KIM and WIN plants (Fig. 3B), and was about 2.5 days longer than on $\mathrm{OH}$ and CYR plants.

Plant population also had a significant effect on adult dry mass of the parasitoid, C. rubecula, $\left(F_{3,230}=13.9, P\right.$ $<0.001$; Fig. 4A), but not on development time from egg to adult $\left(F_{3,230}=1.89, P=0.13\right.$; Fig. $\left.4 \mathrm{~B}\right)$. Wasps, both males and females, emerging from $P$. rapae caterpillars reared on CYR plants were heavier than wasps reared on the KIM population. Weights of adult wasps reared on $\mathrm{OH}$ and WIN plants were not significantly different from wasps reared on either CYR or KIM plants (Fig. 4A). The relationship between fresh mass and dry mass of adult wasps was highly significant (males $F_{1,21}=438, P<0.001, r^{2}=0.95$; females, $\left.F_{1,32}=368, P<0.001, r^{2}=0.92\right)$. Thus, fresh mass accurately approximates body mass of $C$. rubecula. Furthermore, males were smaller $\left(F_{1,230}=332, P<\right.$ 0.001 ; Fig. $4 \mathrm{~A})$ and developed faster $\left(F_{1,230}=47.7, P<\right.$ 0.001 ; Fig. 4B) than female wasps.
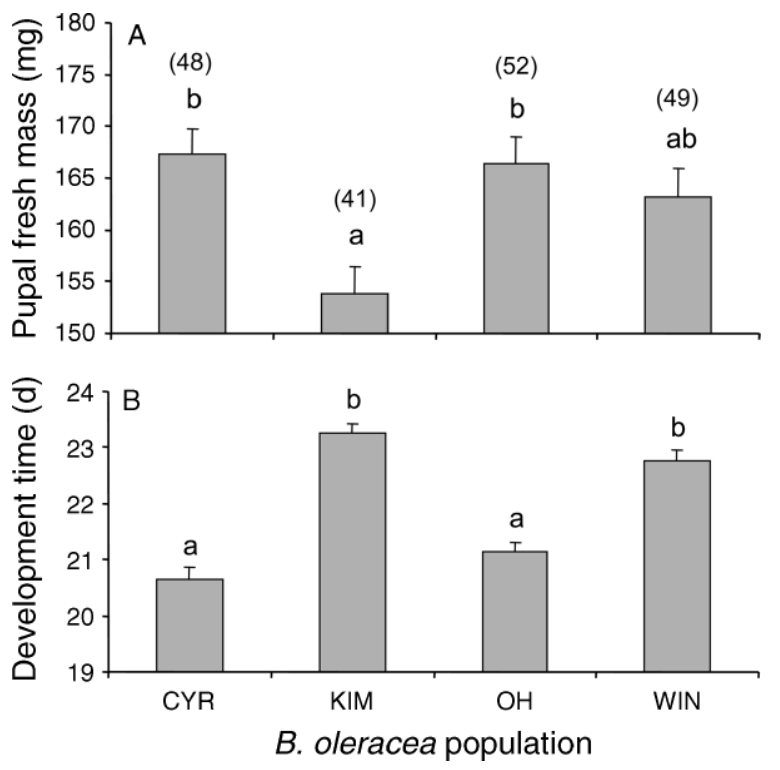

FIG. 3. (A) Pupal fresh mass, and (B) development time from egg to pupa of Pieris rapae that were reared in a greenhouse on either a cultivar (CYR) or on one of three different wild populations (KIM, OH, and WIN) of B. oleracea from the United Kingdom (Dorset). Values are means + SE; bars with the same lowercase letter are not significantly different (Tukey-Kramer test for multiple comparisons among means with $\alpha=0.05)$. Numbers within parentheses denote the number of individuals $(n)$.

Mamestra brassicae-Microplitis mediator.-Survival of the generalist herbivore, M. brassicae, was greatly affected by plant population (Fig. 5). Individual survival curves were all significantly different from each other except for the CYR and KIM curves. None of the larvae reared on the WIN population survived, whereas about $60 \%$ of the larvae reared on $\mathrm{OH}$ and about $80 \%$ of the larvae reared on CYR and KIM plants successfully pupated. Many larvae disappeared during the bioassay. We assumed that these larvae had been cannibalized, since dead ones were also found; furthermore, the larvae could not escape from the petri dishes. Mortality due to cannibalism was much higher on plants of poor food quality. On $\mathrm{OH}$ and WIN plants, $17.5 \%$ and $57.3 \%$ of the larvae died due to cannibalism, whereas this rate was $<2 \%$ on CYR and KIM plants.

Plant population also had a significant effect on pupal biomass $\left(F_{2,221}=66.0 P<0.001\right.$; Fig. $\left.6 \mathrm{~A}\right)$ as well as development time from egg to pupa $\left(F_{2,221}=156, P<\right.$ 0.001; Fig. 6B). Pupal weights were highest when the larvae were reared on CYR plants, intermediate when reared on KIM plants, and lowest when reared on $\mathrm{OH}$ plants (Fig. 6A). Larval development rates were equivalent on CYR and KIM, but caterpillars took 14 days longer to develop into pupae on OH plants (Fig. $6 \mathrm{~B})$.

As in the herbivore, survival of its parasitoid, $M$. mediator, was greatly affected by plant population. Since almost all first-instar caterpillars died when reared on 

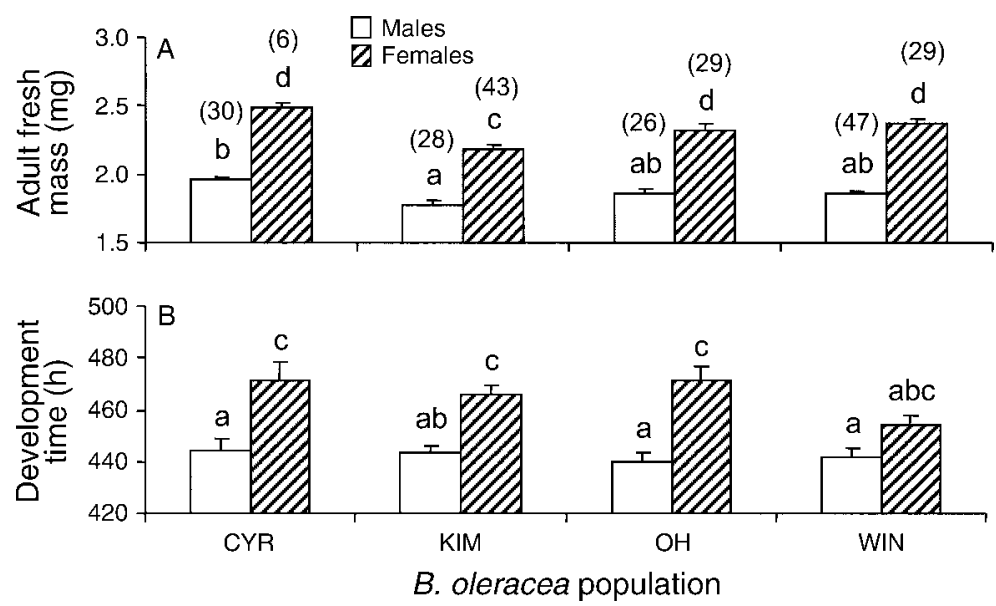

FIG. 4. (A) Adult fresh mass and (B) development time from egg to adult of Cotesia rubecula that were reared on $P$. rapae in a greenhouse on either a cultivar (CYR) or on one of three different wild populations (KIM, OH, and WIN) of B. oleracea from the United Kingdom (Dorset). Results are given separately for males and females. Values are means + SE; bars with the same lowercase letter are not significantly different (Tukey-Kramer test for multiple comparisons among means with $\alpha=0.05$ ). Numbers within parentheses denote the number of individuals $(n)$.

intact WIN plants, this population was not used for experiments with $M$. mediator. The population on which the host developed had a significant effect on survival of the parasitoids $\left(\chi^{2}=7.19, \mathrm{df}=2, P=0.03\right)$. Survival of $M$. mediator was the highest (i.e., $21.6 \%$ ) when the host, M. brassicae, was reared on CYR plants. Survival was $16 \%$ on KIM, whereas only $4 \%$ of the parasitized larvae developed into adults on $\mathrm{OH}$. Since the number of surviving adult wasps was very low, especially the number of females, data on development time and biomass are not presented.

\section{Regression analyses of glucosinolate concentrations and herbivore development}

Regression analyses revealed only a significant positive linear relationship between neoglucobrassicin concentration and development time of P. rapae (Fig. 7A). For M. brassicae, a significant negative relationship was found between concentrations of gluconapin, sinigrin, and total GS, respectively, and survival of the larvae (Fig. 7B-D).

\section{DisCUSSION}

\section{Glucosinolate levels in different populations of Brassica oleracea}

The results of this study show that there was significant variation in GS levels in plants from three wild populations and one cultivar of $B$. oleracea reared under standardized laboratory conditions. Earlier work similarly reported significant differences in GS concentrations among several different wild populations of this species from the same sites (Mithen et al. 1995, Moyes et al. 2000). Previous studies with the WIN and KIM populations (Mithen et al. 1995, Moyes et al. 2000) reported higher levels of aliphatic GS in leaf tissues collected directly from plants in the field than we observed in plants reared in the greenhouse. Levels of the indole GS in those studies were similar to the levels we recorded in herbivore-damaged plants. The differences in GS patterns between the two populations were the same (i.e., the KIM population produced much

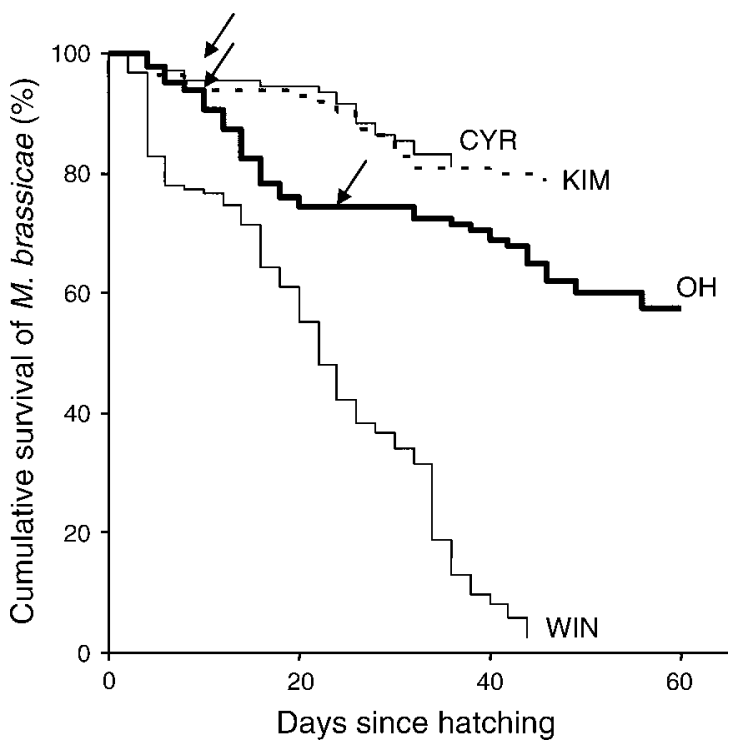

FIg. 5. Cumulative survival tendencies of Mamestra brassicae larvae when reared on detached leaves from either a cultivar (CYR) or from one of three different wild populations (KIM, OH, and WIN) of B. oleracea from the United Kingdom (Dorset). The arrows indicate the time point at which the numbers of larvae were reduced and the analysis was adjusted accordingly (censored data). All pairwise comparisons (logrank test with Bonferroni-corrected $\alpha$ 's) of survival curves were significantly different except for the CYR-KIM comparison $(P$ $=0.76$ ). For other pairwise comparisons: $\mathrm{CYR}-\mathrm{OH}, P=0.02$; OH-KIM, $P=0.008$; WIN-KIM, $P<0.001$; WIN-OH, $P<$ $0.001 ;$ WIN-CV, $P<0.001$. 

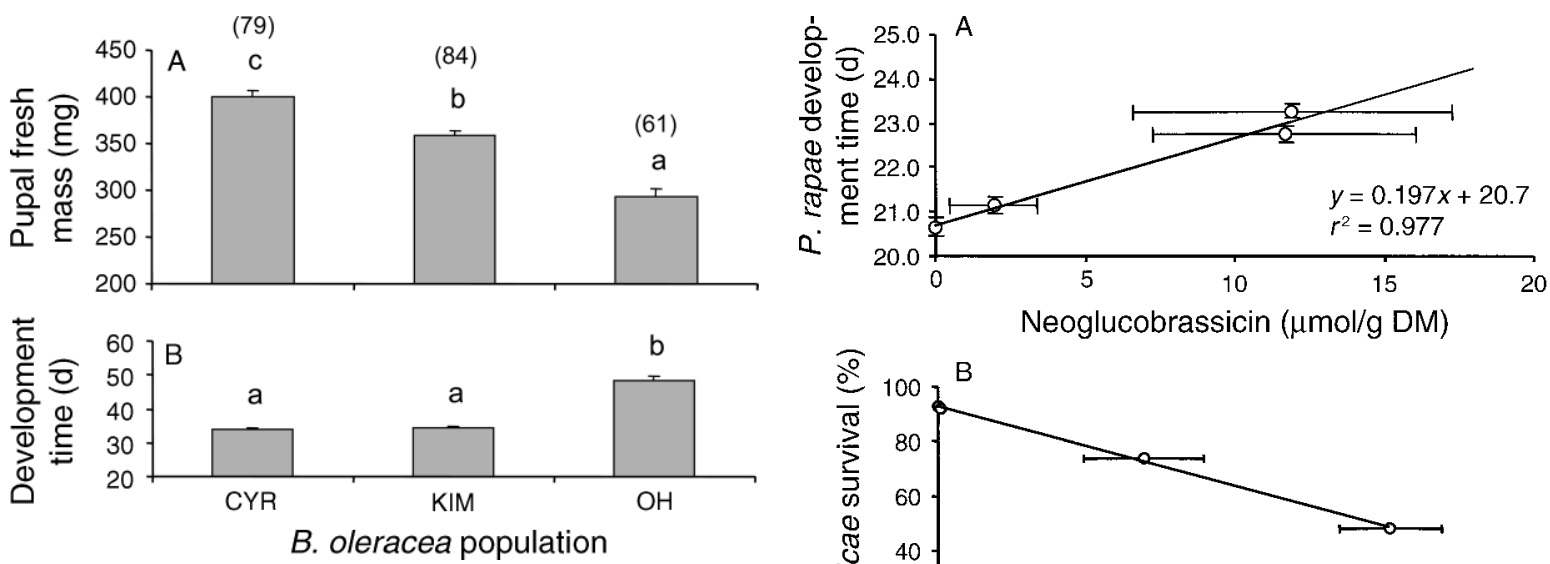

FIG. 6. (A) Pupal fresh mass and (B) development time from egg to pupa of Mamestra brassicae that were reared in a greenhouse on either a cultivar (CYR), or on one of three different wild populations (KIM, OH, and WIN) of B. oleracea from the United Kingdom (Dorset). No data are shown for the WIN population, because all larvae perished before pupation. Values are means + SE; bars with the same lowercase letter are not significantly different (Tukey-Kramer test for multiple comparisons among means with $\alpha=0.05$ ). Numbers within parentheses denote the number of individuals $(n)$.

lower concentrations of aliphatic GS than the WIN population). The observed differences in chemical profiles between plants in the studies by Mithen et al. (1995), Moyes et al. (2000), and this study may be due to concomitant differences in ambient conditions in which the plants were grown (field vs. greenhouse). Furthermore, the field plants may have been older than the plants used here, bearing in mind that some of the $B$. oleracea plants in the Dorset populations exceed 20 years of age (Mitchell and Richards 1979), and GS levels decline with leaf age (Shelton 2005, Gols et al. 2007).

Feeding damage from Pieris rapae differentially affected levels of individual GS. Concentrations of the two indole GS, glucobrassicin and neoglucobrassicin, increased dramatically in response to $P$. rapae feeding on the wild populations, whereas levels of aliphatic GS remained the same as in undamaged plants. Different biosynthetic pathways are involved in the production of aliphatic and indole GS (Halkier and Gershenzon 2006), which may explain the differential effect of herbivore feeding on the expression of these GS. Fatouros et al. (2005) reported that CYR plants treated with regurtitant of parasitized $P$. rapae emitted a different volatile blend than plants treated with regurtitant of unparasitzed caterpillars. We showed that the expression of GS did not differ between plants damaged by parasitized and unparasitized $P$. rapae.

\section{Insect development on different \\ Brassica oleracea populations}

We found that the development of the insect herbivores differed significantly across the different populations of $B$. oleracea. The development of the
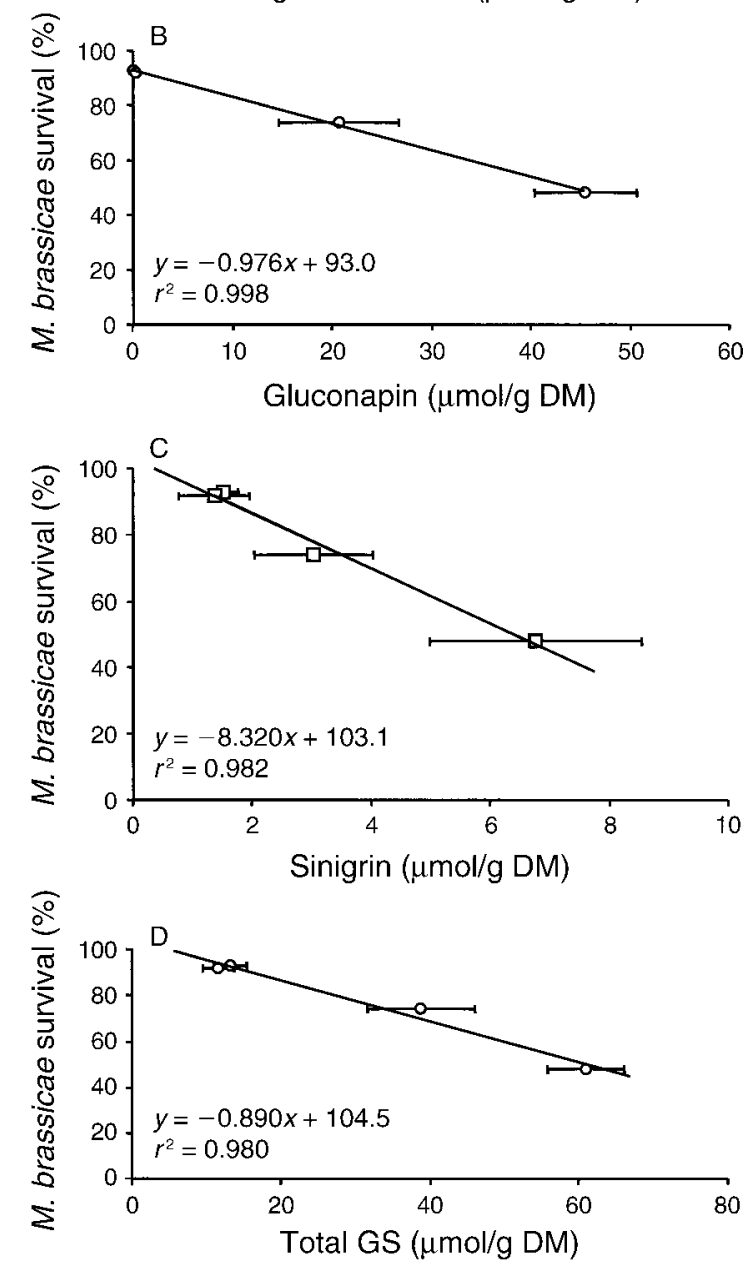

FIG. 7. Relationship between mean concentrations of three GS compounds and for total GS pooled from four different $B$. oleracea populations and mean fitness parameters of two herbivores, $P$. rapae and $M$. brassicae. A significant linear relationship was found for neoglucobrassicin $\left(F_{1,2}=84.1, P=\right.$ $0.01)$ and $P$. rapae development time, as well as for gluconapin $\left(F_{1,2}=1150, P<0.001\right)$, sinigrin $\left(F_{1,2}=112, P=0.009\right)$, and total GS $\left(F_{1,2}=100, P=0.01\right)$, respectively, and survival of $M$. brassicae. GS concentrations (mean \pm SE) were measured halfway through the larval development of the herbivores. For $P$. rapae, which was reared on intact plants, GS concentrations were based on samples $(n=5)$ taken from plants from each population that had been fed upon by $P$. rapae caterpillars for seven days. Mamestra brassicae was reared on detached leaves; therefore, GS concentrations for M. brassicae were determined in undamaged plants $(n=5)$ that were sampled three weeks after oviposition. For survival of $M$. brassicae we used the percentage of caterpillars that were still alive at day 22 on each of the four plant populations. Least-square regression equations are given in the graphs. 
specialist herbivore $P$. rapae was much less negatively affected than that of the generalist herbivore Mamestra brassicae. Specialist herbivores of plants in the Brassicaceae, such as $P$. rapae, have evolved a specific detoxification system to convert the production of deleterious GS breakdown products such as isothiocyanates into less harmful compounds (Ratzka et al. 2002, Wittstock et al. 2004). For example, P. rapae produces an enzyme, a so-called nitrile-specifier protein, which redirects the hydrolysis pathway of GS from isothiocyanates into the production of less toxic nitriles (Wittstock et al. 2004). Here we show that the performance of $P$. rapae was differentially affected by $B$. oleracea populations that differ in their GS profile. Regression analyses revealed that $P$. rapae developed most poorly on plants from the KIM and WIN population, which contained the highest levels of the indole GS, neoglucobrassicin. These two populations differed considerably with respect to other GS concentrations, suggesting that neoglucobrassicin affects plant quality for this herbivore species and that detoxification of this GS may be less efficient.

The role of indole GS in defense against insect herbivory is poorly understood, in part, because levels of indole GS in foliage of cultivated B. oleracea and other Brassicaceous species are much lower than in wild B. oleracea plants (Giamoustaris and Mithen 1995, Rosa 1999, Traw 2002). Only the hydrolysis products of GS, such as isothiocyanates, have been shown to be active against insects (Agrawal and Kurashige 2003, Wittstock et al. 2004). These products are formed after tissue damage, followed by myrosinase enzyme activity. Hydrolysis of aliphatic GS results in the formation of stable isothiocyanates. Attempts to isolate stable isothiocyanate products of indole GS have not been successful (Bones and Rossiter 2006), suggesting that hydrolysis products of indole GS undergo further degradation. Whether indole GS and their breakdown products are detoxified by insect herbivores specialized on the Brassicaceae in the same way as aliphatic GS is unknown and merits further investigation.

For M. brassicae, regression analyses revealed a significant negative relationship between concentrations of the two aliphatic GS, sinigrin and gluconapin, and herbivore survival. M. brassicae developed most poorly on WIN and OH plants, which contained not only high levels of sinigrin and gluconapin, but also high overall GS levels. We also found a negative relationship between total levels of GS and survival of M. brassicae larvae, suggesting that $M$. brassicae responds to high levels of GS rather than to specific GS. Furthermore, $M$. brassicae was reared on detached leaves, whereas $P$. rapae was reared on intact plants. Further research is necessary to reveal the time course of GS induction. The induction of indole GS may not have reached similar levels in detached leaves as in intact plants and this may have influenced the development of $M$. brassicae.
The development of the two parasitoid species also differed across the various populations of $B$. oleracea. Most importantly, parasitoid development and survival appeared to closely reflect the effects of plant quality on host development. Various studies have similarly reported that the performance of both herbivores and their natural enemies are negatively affected by plant allelochemicals (Campbell and Duffey 1979, Barbosa et al. 1986, Gunasena et al. 1990, Sznajder and Harvey 2003, Harvey 2005, Harvey et al. 2007).

Little is known about the means by which parasitoid larvae deal with allelochemicals in host diet that may diffuse through the gut wall and accumulate in host hemolymph or fat body. Endoparasitoids may be particularly susceptible to plant toxins because the alimentary tract of parasitoid larvae is not externally connected until after the parasitoid disposes of host tissues and constructs a cocoon, into which the meconium is voided (Quicke 1997). Barbosa et al. (1986) and Bowers (2003) found traces of nicotine and catalpol in the larval by-products (cocoon silk, meconium) of the parasitoid Cotesia congregata developing in two of its hosts, Manduca sexta and Ceratomia catalpae, respectively. It is likely that the ability of parasitoids to deal with plant allelochemicals is largely dependent on the degree to which they are exposed to them, and suggests that species such as Cotesia rubecula probably are well-adapted to deal with high levels of GS in host diet (see also Harvey et al. 2003).

\section{Evolution of plant defenses in natural populations of Brassica oleracea}

Although plant defenses have been well-studied in a multitrophic framework, and the effects of secondary chemistry on herbivores and their natural enemies are well-documented (see reviews by Harvey 2005, Ode 2006), the factors underlying the genetic variation in plant defense in natural populations are virtually unknown. Several studies have investigated spatial and temporal variation in plant chemistry over broad geographical scales (Agrawal 2005, Zangerl and Berenbaum 2005). The cabbage populations studied here all grow within $15 \mathrm{~km}$ of one another, and perhaps more striking is the fact that the two most different populations in terms of defense chemistry (WIN and KIM) are separated by $<5 \mathrm{~km}$. Notably, the KIM plants grow atop cliffs that are fully exposed to strong prevailing westerly winds, whereas the WIN population occurs in a very sheltered valley. Although they have not been measured, it is almost certain that important microclimatic variables, such as wind speed, temperature, and access to light and water differ markedly between the different coastal populations of B. oleracea. This variation in abiotic traits may be linked with biotic selection pressures, such as herbivory and the efficacy of natural enemies, such as parasitoids.

Because we studied interactions involving only single pairs of generalist and specialist herbivores and their 
endoparasitoids, these results cannot be used to predict differential herbivore pressures in natural populations of B. oleracea. It is known that herbivory may be a major factor in driving the evolution of direct plant defenses over comparatively large spatial scales (Zangerl and Berenbaum 2005). However, little is currently known about the factors that maintain the dramatic differences in secondary chemistry of the wild B. oleracea populations. Studies are planned in which the population dynamics of both herbivores and parasitoids will be examined in different wild populations of $B$. oleracea growing along the British coastline. These studies are aimed at elucidating a suite of biotic and abiotic factors that mediate the variation in secondary plant chemistry in B. oleracea.

\section{AcKNowledgments}

We thank Leo Koopman and André Gidding for the rearing of insects, and Ciska Raaijmakers for assistance with the glucosinolate analyses. Glucobrassicin was kindly provided by M. Reichelt, Max Planck Institute for Chemical Ecology, Jena, Germany.

\section{Literature Cited}

Agerbirk, N., C. E. Olsen, and J. K. Nielsen. 2001. Seasonal variation in leaf glucosinolates and insect resistance in two types of Barbarea vulgaris ssp. arcuata. Phytochemistry 58: 91-100.

Agrawal, A. A. 2005. Natural selection on common milkweed (Asclepias syriaca) by a community of specialized insect herbivores. Evolutionary Ecology Research 7:651-667.

Agrawal, A. A., J. K. Conner, M. T. J. Johnson, and R. Wallsgrove. 2002. Ecological genetics of an induced plant defense against herbivores: additive genetic variance and costs of phenotypic plasticity. Evolution 56:2206-2213.

Agrawal, A. A., and N. S. Kurashige. 2003. A role for isothiocyanates in plant resistance against the specialist herbivore Pieris rapae. Journal of Chemical Ecology 29: 1403-1415.

Arthur, A. P., and P. G. Mason. 1986. Life-history and immature stages of the parasitoid Microplitis mediator (Hymenoptera, Braconidae), reared on the bertha armyworm Mamestra configurata (Lepidoptera, Noctuidae). Canadian Entomologist 118:487-491.

Barbosa, P., P. Gross, and J. Kemper. 1991. Influence of plant allelochemicals on the tobacco hornworm and its parasitoid, Cotesia congregata. Ecology 72:1567-1575.

Barbosa, P., J. A. Saunders, J. Kemper, R. Trumbule, J. Olechno, and P. Martinat. 1986. Plant allelochemicals and insect parasitoids: effects of nicotine on Cotesia congregata (Say) (Hymenoptera, Braconidae) and Hyposoter annulipes (Cresson) (Hymenoptera, Ichneumonidae). Journal of Chemical Ecology 12:1319-1328.

Benrey, B., and R. F. Denno. 1997. The slow growth-high mortality hypothesis: a test using the cabbage butterfly. Ecology 78:987-999.

Blau, P. A., P. Feeny, L. Contardo, and D. S. Robson. 1978. Allylglucosinolate and herbivorous caterpillars: a contrast in toxicity and tolerance. Science 200:1296-1298.

Bodnaryk, R. P. 1992. Effects of wounding on glucosinolates in the cotyledons of oilseed rape and mustard. Phytochemistry 31:2671-2677.

Bones, A. M., and J. T. Rossiter. 2006. The enzymic and chemically induced decomposition of glucosinolates. Phytochemistry 67:1053-1067.
Bowers, M. D. 1983. The role of iridoid glycosides in host-plant specificity of checkerspot butterflies. Journal of Chemical Ecology 9:475-493.

Bowers, M. D. 2003. Host plant suitability and defensive chemistry of the Catalpa sphinx, Ceratomia catalpae. Journal of Chemical Ecology 29:2359-2367.

Campbell, B. C., and S. S. Duffey. 1979. Tomatine and parasitic wasps: potential incompatibility of plant antibiosis with biological control. Science 205:700-702.

Carter, D. J. 1984. Pest Lepidoptera of Europe with special reference to the British Isles. Junk, Dordrecht, The Netherlands.

European Community. 1990. Oil seeds-determination of glucosinolates high performance liquid chromatography. Official Journal of the European Communities L 170/28. Annex VIII:03.07.:27-34.

Fatouros, N. E., J. J. A. Van Loon, K. A. Hordijk, H. M. Smid, and M. Dicke. 2005. Herbivore-induced plant volatiles mediate in-flight host discrimination by parasitoids. Journal of Chemical Ecology 31:2033-2047.

Giamoustaris, A., and R. Mithen. 1995. The effect of modifying the glucosinolate content of leaves of oilseed rape (Brassica napus ssp. oleifera) on its interaction with specialist and generalist pests. Annals of Applied Biology 126:347-363.

Gols, R., C. E. Raaijmakers, N. M. van Dam, M. Dicke, T. Bukovinszky, and J. A. Harvey. 2007. Temporal changes affect plant chemistry and tritrophic interactions. Basic and Applied Ecology 8:421-433.

Gripenberg, S., and T. Roslin. 2007. Up or down in space? Uniting the bottom-up versus top-down paradigm and spatial ecology. Oikos 116:181-188.

Gunasena, G. H., S. B. Vinson, and H. J. Williams. 1990. Effects of nicotine on growth, development, and survival of the tobacco budworm (Lepidoptera, Noctuidae) and the parasitoid Campoletis sonorensis (Hymenoptera, Ichneumonidae). Journal of Economic Entomology 83:1777-1782.

Hairston, N. G., F. E. Smith, and L. B. Slobodkin. 1960. Community structure, population control, and competition. American Naturalist 94:421-425.

Halkier, B. A., and J. Gershenzon. 2006. Biology and biochemistry of glucosinolates. Annual Review of Plant Biology 57:303-333.

Harvey, J. A. 2005. Factors affecting the evolution of development strategies in parasitoid wasps: the importance of functional constraints and incorporating complexity. Entomologia Experimentalis et Applicata 117:1-13.

Harvey, J. A., M. A. Jervis, R. Gols, N. Q. Jiang, and L. E. M. Vet. 1999. Development of the parasitoid, Cotesia rubecula (Hymenoptera: Braconidae) in Pieris rapae and Pieris brassicae (Lepidoptera: Pieridae): evidence for host regulation. Journal of Insect Physiology 45:173-182.

Harvey, J. A., N. M. van Dam, and R. Gols. 2003. Interactions over four trophic levels: foodplant quality affects development of a hyperparasitoid as mediated through a herbivore and its primary parasitoid. Journal of Animal Ecology 72: $520-531$.

Harvey, J. A., N. M. van Dam, L. M. A. Witjes, R. Soler, and R. Gols. 2007. Effects of dietary nicotine on the development of an insect herbivore, its parasitoid and secondary hyperparasitoid over four trophic levels. Ecological Entomology 32:15-23.

Karban, R., and I. T. Baldwin. 1997. Induced responses to herbivory. University of Chicago Press, Chicago, Illinois, USA.

Keeler, M. S., F. S. Chew, B. C. Goodale, and J. M. Reed. 2006. Modelling the impacts of two exotic invasive species on a native butterfly: top-down vs. bottom-up effects. Journal of Animal Ecology 75:777-788.

Kleinbaum, D. G. 1996. Survival analysis: a self-learning text. Springer-Verlag, New York, New York, USA. 
Li, Q., S. D. Eigenbrode, G. R. Stringham, and M. R. Thiagarajah. 2000. Feeding and growth of Plutella xylostella and Spodoptera eridania on Brassica juncea with varying glucosinolate concentrations and myrosinase activities. Journal of Chemical Ecology 26:2401-2419.

Magrath, R., C. Herron, A. Giamoustaris, and R. Mithen. 1993. The inheritance of aliphatic glucosinolates in Brassica napus. Plant Breeding 111:55-72.

Mitchell, N. D., and A. J. Richards. 1979. Biological flora of the British Isles-Brassica oleracea L. ssp. oleracea ( $B$. sylvestris (L.) Miller). Journal of Ecology 67:1087-1096.

Mithen, R., A. F. Raybould, and A. Giamoustaris. 1995. Divergent selection for secondary metabolites between wild populations of Brassica oleracea and its implications for plant-herbivore interactions. Heredity 75:472-484.

Moyes, C. L., H. A. Collin, G. Britton, and A. E. Raybould. 2000. Glucosinolates and differential herbivory in wild populations of Brassica oleracea. Journal of Chemical Ecology 26:2625-2641.

Moyes, C. L., and A. F. Raybould. 2001. The role of spatial scale and intraspecific variation in secondary chemistry in host-plant location by Ceutorhynchus assimilis (Coleoptera: Curculionidae). Proceedings of the Royal Society of London Series B 268:1567-1573.

Nishida, R. 2002. Sequestration of defensive substances from plants by Lepidoptera. Annual Review of Entomology 47: 57-92.

Ode, P. J. 2006. Plant chemistry and natural enemy fitness: effects on herbivore and natural enemy interactions. Annual Review of Entomology 51:163-185.

Orr, D. B., and D. J. Boethel. 1986. Influence of plant antibiosis through four trophic levels. Oecologia 70:242-249.

Quicke, D. L. J. 1997. Parasitic wasps. Cambridge University Press, Cambridge, UK.

Rask, L., E. Andreasson, B. Ekbom, S. Eriksson, B. Pontoppidan, and J. Meijer. 2000. Myrosinase: gene family evolution and herbivore defense in Brassicaceae. Plant Molecular Biology 42:93-113.

Ratzka, A., H. Vogel, D. J. Kliebenstein, T. Mitchell-Olds, and J. Kroymann. 2002. Disarming the mustard oil bomb. Proceedings of the National Academy of Sciences (USA) 99:11223-11228.

Raybould, A. F., R. J. Mogg, R. T. Clarke, C. J. Gliddon, and A. J. Gray. 1999. Variation and population structure at microsatellite and isozyme loci in wild cabbage (Brassica oleracea L.) in Dorset (UK). Genetic Resources and Crop Evolution 46:351-360.

Renwick, J. A. A. 2002. The chemical world of crucivores: lures, treats and traps. Entomologia Experimentalis et Applicata 104:35-42.

Rosa, E. A. S. 1999. Chemical composition. Pages 315-357 in C. Gomez-Campo, editor. Developments in plant genetics and breeding. 4. Biology of Brassica coenospecies. Elsevier Science, Amsterdam, The Netherlands.
Rosenthal, G. A., and M. R. Berenbaum. 1992. Herbivores: their interactions with secondary plant metabolites. Academic Press, San Diego, California, USA.

Roth, S., C. Knorr, and R. L. Lindroth. 1997. Dietary phenolics affects performance of the gypsy moth (Lepidoptera: Lymantriidae) and its parasitoid Cotesia melanoscela (Hymenoptera: Braconidae). Environmental Entomology 26: 668-671.

SAS Institute. 1999-2001. SAS statistical software. Version 8.02. SAS Institute, Cary, North Carolina, USA.

Schoonhoven, L. M., J. J. A. van Loon, and M. Dicke. 2005. Insect-plant biology. Second edition. Oxford University Press, Oxford, UK.

Shelton, A. L. 2005. Within-plant variation in glucosinolate concentrations of Raphanus sativus across multiple scales. Journal of Chemical Ecology 31:1711-1732.

Sznajder, B., and J. A. Harvey. 2003. Second and third trophic level effects of differences in plant species reflect dietary specialisation of herbivores and their endoparasitoids. Entomologia Experimentalis et Applicata 109:73-82.

Theunissen, J., H. den Uuden, and A. K. H. Wit. 1985. Feeding capacity of caterpillars on cabbage, a factor in crop loss assessment. Entomologia Experimentalis et Applicata 39: 255-260.

Traw, M. B. 2002. Is induction response negatively correlated with constitutive resistance in black mustard? Evolution 56: 2196-2205.

Traw, M. B., and T. E. Dawson. 2002. Reduced performance of two specialist herbivores (Lepidoptera: Pieridae, Coleoptera: Chrysomelidae) on new leaves of damaged black mustard plants. Environmental Entomology 31:714-722.

van Dam, N. M., K. Hadwich, and I. T. Baldwin. 2000. Induced responses in Nicotiana attenuata affect behavior and growth of the specialist herbivore Manduca sexta. Oecologia 122:371-379.

van Dam, N. M., L. Witjes, and A. Svatos. 2004. Interactions between aboveground and belowground induction of glucosinolates in two wild Brassica species. New Phytologist 161: 801-810.

Williams, I. S. 1999. Slow-growth, high-mortality - a general hypothesis, or is it? Ecological Entomology 24:490-495.

Wittstock, U., N. Agerbirk, E. J. Stauber, C. E. Olsen, M. Hippler, T. Mitchell-Olds, J. Gershenson, and H. Vogel. 2004. Successful herbivore attack due to metabolic diversion of a plant chemical defense. Proceedings of the National Academy of Sciences (USA) 101:4859-4864.

Zangerl, A. R., and M. R. Berenbaum. 1993. Plant chemistry, insect adaptations to plant chemistry, and host plant utilization patterns. Ecology 74:47-54.

Zangerl, A. R., and M. R. Berenbaum. 2005. Increase in toxicity of an invasive weed after reassociation with its coevolved herbivore. Proceedings of the National Academy of Sciences (USA) 102:15529-15532.

\section{APPENDIX A}

ANOVA tables for ln-transformed glucosinolate concentrations in leaf samples from four different Brassica oleracea populations (Ecological Archives E089-096-A1)

\section{APPENDIX B}

ANOVA tables for pupal/adult mass and development time for the herbivores Mamestra brassicae and Pieris rapae, and the parasitoid Cotesia rubecula when reared on four different Brassica oleracea populations (Ecological Archives E089-096-A2). 